\title{
Chromatin dynamics during interphase explored by single-particle tracking
}

\author{
Valeria Levi ${ }^{1} \&$ Enrico Gratton ${ }^{2 *}$ \\ ${ }^{1}$ Laboratorio de Electrónica Cuántica, Departamento de Física, Universidad de Buenos Aires, \\ Pabellón I Ciudad Universitaria, 1428, Buenos Aires, Argentina; ${ }^{2}$ Laboratory for Fluorescence Dynamics, \\ University of California at Irvine, Rockwell Engineering Center \#204, Irvine, CA, 92697-2715, USA; \\ Tel: +1-949-8247085; Fax: +1-949-8241727; E-mail: lfd@uci.edu \\ * Correspondence
}

Key words: chromatin dynamics, single-particle tracking, two-photon microscopy

\begin{abstract}
Our view of the structure and function of the interphase nucleus has changed drastically in recent years. It is now widely accepted that the nucleus is a well organized and highly compartmentalized organelle and that this organization is intimately related to nuclear function. In this context, chromatin-initially considered a randomly entangled polymer - has also been shown to be structurally organized in interphase and its organization was found to be very important to gene regulation. Relevant and not completely answered questions are how chromatin organization is achieved and what mechanisms are responsible for changes in the positions of chromatin loci in the nucleus. A significant advance in the field resulted from tagging chromosome sites with bacterial operator sequences, and visualizing these tags using green fluorescent protein fused with the appropriate repressor protein. Simultaneously, fluorescence imaging techniques evolved significantly during recent years, allowing observation of the time evolution of processes in living specimens. In this context, the motion of the tagged locus was observed and analyzed to extract quantitative information regarding its dynamics. This review focuses on recent advances in our understanding of chromatin dynamics in interphase with the emphasis placed on the information obtained from single-particle tracking (SPT) experiments. We introduce the basis of SPT methods and trajectory analysis, and summarize what has been learnt by using this new technology in the context of chromatin dynamics. Finally, we briefly describe a method of SPT in a twophoton excitation microscope that has several advantages over methods based on conventional microscopy and review the information obtained using this novel approach to study chromatin dynamics.
\end{abstract}

$\begin{array}{ll}\text { Abbreviations } \\ \text { CHO } & \text { Chinese hamster ovary cell line } \\ \text { DAPI } & \text { 4,6-diamidino-2-phenylindole } \\ \text { DHFR } & \text { dihydrofolate reductase } \\ \text { EGFP } & \text { enhanced green fluorescent protein } \\ \text { GFP } & \text { green fluorescent protein } \\ \text { IR } & \text { infrared } \\ \text { MSD } & \text { mean square displacement } \\ \text { NA } & \text { numerical aperture }\end{array}$

\section{Abbreviations}

$\mathrm{CHO}$

DAPI

DHFR

EGFP

GFP

MSD

NA $\begin{array}{ll}\text { S/N ratio } & \text { signal-to-noise ratio } \\ \text { SPT } & \text { single-particle tracking }\end{array}$

Chromatin organization in the interphase nucleus

Chromatin organization in interphase nuclei has been a subject of great interest over the last decade (Heard $\&$ Bickmore 2007). First results pointing toward a 
compartmentalization of the interphase nucleus were intuitively striking because the nucleus does not contain compartments limited by membranes as do organelles in the cell cytoplasm. It is now widely accepted that nuclear substructures constitute compartments in which specific functions are performed (reviewed in Dundr \& Misteli 2001, Handwerger \& Gall 2006).

Similarly, the idea that chromatin was organized in defined regions later known as chromosomes territories was unexpected. Even current textbooks teach us that chromatin is a polymer randomly entangled in the interphase nucleus (see references in Cremer \& Cremer 2006a,b). Although a model of territorial organization of chromatin in interphase was initially proposed by Rabl and Boveri in the early 20th century (for a recent review see Cremer \& Cremer 2006a,b), several years had to pass before new tools in microscopy and cell biology allowed experimental demonstration of the existence of chromosome territories (see for example, Cremer et al. 1982, Lichter et al. 1988, Branco \& Pombo 2006).

These studies demonstrated that chromosomes in interphase cells occupy well-defined volumes in the nucleus; moreover, they have preferred positions relative to the nuclear membrane and with respect to each other.

The internal architecture of these territories is also not random: some studies showed that gene-rich and gene-poor sequences are mostly localized in the periphery and in the core of the chromosome territory, respectively (Kurz et al. 1996). These results leaded to a model proposing that the transcription machinery is located in interchromatin domains, i.e. regions between chromosomes territories. According to this model, active genes are mainly located in the surface of these territories so as to access the transcription and splicing machineries. This model was further supported by studies showing that genes may move to the interchromatin region in response to transcription (Volpi et al. 2000, Chambeyron et al. 2005).

However, the architecture of chromatin in interphase seems to be more complex and there are no general rules that can be applied to every chromosome territory in every cell line. Contrary to the proposed models, coding sequences were found in the interior of chromatin territories which do not change their positions during gene expression (Mahy et al. 2002). Also, transcription sites were found to be located all over chromatin territories (Abranches et al. 1998, Verschure et al. 1999, Branco \& Pombo 2006), in contrast to the proposition of transcription taking place only at interchromatin regions. Moreover, the nuclear periphery contains silencing compartments (Andrulis et al. 1998) as well as activating compartments. Contrary to the observations in mammalian cells, nuclear pores in Saccharomyces cerevisiae have been identified as important sites of transcription (Brickner \& Walter 2004, Menon et al. 2005, Schmid et al. 2006) and, in some instances, transcription activation is accompanied by motion of the sequence toward the nuclear periphery (Brickner \& Walter 2004).

A significant amount of evidence has been accumulated in recent years pointing toward a model in which chromatin territories are interspersed with interchromatin domains. To activate transcription, in some cases specific local reorganization of regions of these territories occurs (Branco \& Pombo 2006, 2007), while in others the transcription machinery is physically excluded from particular chromatin territories, providing a mechanism for silencing gene expression (Chaumeil et al. 2006).

Two novel technologies, chromosome conformation capture (Dekker et al. 2002) and chromosome conformation capture-on-chip (Simonis et al. 2006) allowed the study of intra- and interchromosomal interactions for the first time and showed the relevance of these interactions to gene activity regulation. In these methods, isolated nuclei are treated with formaldehyde, producing crosslinking of nearby chromatin segments. The crosslinked products are digested with a restriction enzyme and analyzed to quantify the relative frequencies with which different sites of chromatin were crosslinked. Using this technology, Simonis et al. (Simonis et al. 2006) showed that the actively transcribed $\beta$-globin locus in fetal liver interacts with a completely different set of loci from its transcriptionally silent counterpart in brain. While the majority of the $\beta$-globin interacting loci in fetal liver contained actively transcribed genes, most of the interacting loci in brain did not show gene activity.

In conclusion, although we do not have a complete knowledge of the three-dimensional architecture of chromatin in the nucleus, factors such as the relative positioning of genes, regulatory sequences and transcription factors seem to be very important for transcription. It is therefore a priority to investigate 
chromatin organization during interphase to fully understand the mechanisms involved in gene expression regulation.

\section{Studying intracellular dynamics by single-particle tracking}

In recent years, single-particle tracking (SPT) techniques have increasingly been used to study the dynamics of cellular compounds ranging from small lipids and proteins to organelles (for recent reviews see Cheezum et al. 2001, Levi \& Gratton 2007).

These techniques were developed to follow the position of individual particles over time. Provided the spatial and temporal resolution of the method is adequate, these trajectories can be analyzed to extract quantitative information about the mechanism involved in the motion of the particle. Since the properties of the particles are not averaged as in bulk measurements, SPT can easily distinguish populations of particles with different motion properties and recognize changes between different mechanisms of motion, thus allowing the study of complex processes (see for example, Dahan et al. 2003, Lakadamyali et al. 2003). These characteristics make SPT an appealing technique to achieve the ultimate goal of understanding dynamics in cells. The most common methods used to track fluorescent particles are based on recording images of the cells in a wide-field or confocal fluorescence microscope as a function of time and afterward locating the particle of interest in each frame of the stack.

A point-like particle observed through an optical microscope forms a diffraction-limited image of width approximately equal to $\lambda /(2 \mathrm{NA})$, where $\lambda$ is the wavelength of the light and NA is the numerical aperture of the objective (Abramowitz 2003). This diffraction limit implies that the image of the particle would appear to have a diameter of $\sim 200 \mathrm{~nm}$ for visible light, making it impossible to achieve optical resolution below this limit.

Several techniques have been proposed to locate particles with higher precision than that given by the diffraction limit. A common approach is based on the fact that the intensity of the image of a point-like particle follows a diffraction-limited distribution whose center corresponds to the position of the particle. This position can be determined with high precision using an algorithm that finds the center of the distribution. The most common algorithm used to track particles is based on fitting a Gaussian distribution function to the intensity profile of the image (Figure 1A). Using this method, the precision of position determination can be as high as $1.5 \mathrm{~nm}$ (Yildiz et al. 2003) depending on experimental factors such as the number of photons collected per particle, pixel size, and background noise (Thompson et al. 2002).

Once the position of the particle is obtained in every frame of the movie, its trajectory is analyzed to extract quantitative information regarding the mechanism underlying the motion (for a general review see Saxton \& Jacobson 1997). The most common approach to analyzing single-particle trajectories is to calculate the mean square displacement (MSD) as follows:

$$
\begin{aligned}
\operatorname{MSD}(\tau)=\langle & {[x(t)-x(t+\tau)]^{2}+[y(t)-y(t+\tau)]^{2} } \\
& \left.+[z(t)-z(t+\tau)]^{2}\right\rangle
\end{aligned}
$$

where $x, y$ and $z$ are the particle coordinates and $\tau$ is a lag time.

MSD values at longer $\tau$ are calculated with a lower numbers of data; these values thus have lower statistical significance. A common criterion is to restrict the analysis to $\tau$ values lower than $1 / 4$ of the total time of the trajectory (Saxton \& Jacobson 1997).

MSD indicates how far a particle traveled after a time lag. Thus; its dependence on $\tau$ is related to the motion properties of the particle. Consequently, a possible mechanism for the particle motion can be derived by comparing the experimental MSD plot with predictions from different motion models. Figure 1B-D shows MSD plots expected for simple mechanisms of motion such as random diffusion, corralled diffusion, active transport and anomalous subdiffusion (Qian et al. 1991, Saxton 1993, 1994a,b, 1995, 1996, 1997, Saxton \& Jacobson 1997). This last behavior can be the consequence of the binding of the particle to a given fixed target or may be due to the presence of obstacles to free motion.

A common concern when doing tracking experiments in living cells is that regions of the cell may move during the experiment and thus the trajectory of the studied particle will reflect both its intrinsic motion and the motion of the region in which the particle is included. In the case of tracking experiments in the nucleus, different strategies have been 

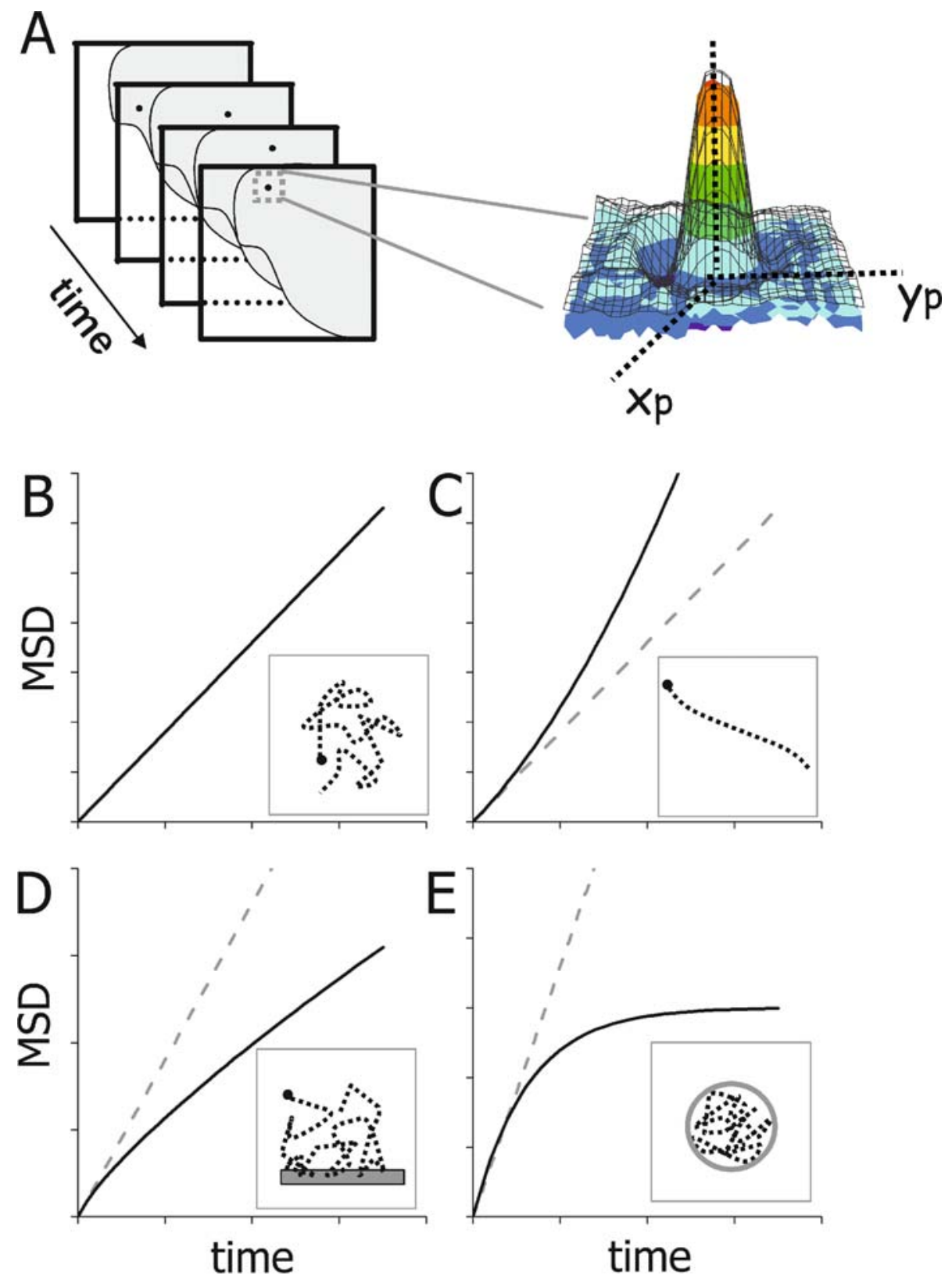

Figure 1. Single-particle tracking. (A) Determination of a particle trajectory by analysis of an image stack. A theoretical distribution function (black grid) is fitted to the image of a point particle in every image (colored in intensity-code scale). The center of the distribution function corresponds to the position of the particle. (B-E) Expected MSD curves for random diffusion (B), active transport (C), anomalous subdiffusion (D) and confined diffusion (E). Dashed lines in each plot represent the behavior expected for a free, randomly diffusing particle. The insets to the plots schematically show trajectories expected for particles moving under these regimes and possible interactions or restrictions that account for these trajectories.

followed to uncouple the motion of the fluorescent particle from the motion of the whole nucleus. Most of them are based on introducing an internal reference, e.g. labeling the nuclear envelope, and studying the motion of the particle relative to the reference (see, for example, Heun et al. 2001, Marshall et al. 1997) 
Another possibility is to use cells whose nuclei are fluorescent and then to align the nucleus in each image before the tracking analysis (see, for example, Chuang et al. 2006); this approach has the disadvantage that nuclear rotations may not be detected.

\section{Chromatin dynamics in the interphase nucleus}

As mentioned above, the spatial organization of chromatin is intimately related to gene expression. Important and not completely answered questions are how the organization of chromatin is achieved and how specific regions change their position in the nucleus.

Most of the techniques used to study chromatin architecture cannot reveal details regarding the dynamics of chromatin, providing only a static view of the nucleus in a given condition. To observe chromatin motion, it is required to use labeling techniques compatible with living cell observation. Initial work made use of DNA-specific fluorescent dyes such as 4,6-diamidino-2-phenylindole (DAPI) (Crissman \& Hirons 1994) and fluorescent nucleotides (Zink et al. 1998). With the emergence of green fluorescent protein (GFP) technology, histones fused to fluorescent protein variants (Kanda et al. 1998) were used to label chromatin.

A significant breakthrough in the field was the development of a novel approach to labeling specific DNA sequences in live cells that consists in the insertion of lac operator repeats at specific chromosome locations. This locus can be detected through the binding of the lac repressor protein fused to GFP and engineered to contain a nuclear localization signal (Robinett et al. 1996, Belmont et al. 1999). The fluorescently labeled sequence is seen as a bright dot in the nucleus, which is dimly fluorescent due to unbound EGFP-Lac repressor. The motion of this bright dot can be followed and the recovered trajectory analyzed to obtain information about the mechanism by which the tagged sequence is moving.

Studies on Saccharomyces cerevisiae (Marshall et al. 1997), mammalian cells (Chubb et al. 2002) and Drosophila spermatocytes (Vazquez et al. 2001) have shown that GFP-tagged chromatin loci undergo Brownian motion limited to a subregion of the nucleus during interphase. However, the motion seems to be more complex than constrained, passive diffusion. Chubb et al. (2002) demonstrated that chromatin associated with the nucleolus or localized in the nuclear periphery is more restricted in its movements than other more nucleoplasmic genomic regions. Rosa et al. (2006) showed that two chromosomal sites exhibiting preferential association with the nuclear membrane, are confined to regions of different size, with the site with higher levels of transcription exploring larger regions. Heun et al. (Heun et al. 2001) reported that early and late origins of replication in yeast are more mobile in $G_{1}$ phase than in $S$ phase. Also, the movement in $G_{1}$ phase was highly sensitive to ATP depletion and to changes in metabolic status. On the other hand, Tumbar \& Belmont (2001) showed that a specific DNA region in $\mathrm{CHO}$ cells changes its position from the nuclear periphery to the center in response to VP16 transcriptional activator.

An important factor that limits the temporal resolution and observation time window in studies of chromatin dynamics is that the motion of the labeled sequence is highly sensitive to photodamage, as was shown in yeast and mammalian cells (Hediger et al. 2004, Chuang et al. 2006). For example, Chuang et al. (2006) showed that the motion of a tagged chromatin sequence changes significantly after taking as few as 10 images when using a mercury lamp as excitation source in regular imaging conditions. Thus, special care has to be taken with respect to the illumination conditions, exposure time, and maximum number of frames that can be acquired.

\section{Two-photon microscopy}

In 1990, Denk, Stricler and Webb invented twophoton excitation microscopy (Denk et al. 1990). This relatively new microscopy is an attractive alternative when dealing with photobleaching and photodamage in wide-field and confocal fluorescence microscopes. Excellent reviews on this technique can be found in the literature (see, for example, So et al. 2000, Svoboda \& Yasuda 2006).

Two-photon excitation is a nonlinear process involving the almost simultaneous absorption of two photons $\left(\sim 10^{-15} \mathrm{~s}\right)$ that in the simplest case will each have half of the energy required for the transition to the excited state (Figure 2A). The probability of this simultaneous absorption increases with the square of the photon flux since two photons have to encounter the fluorophore at almost the same time. Thus, very high photon fluxes are necessary to 

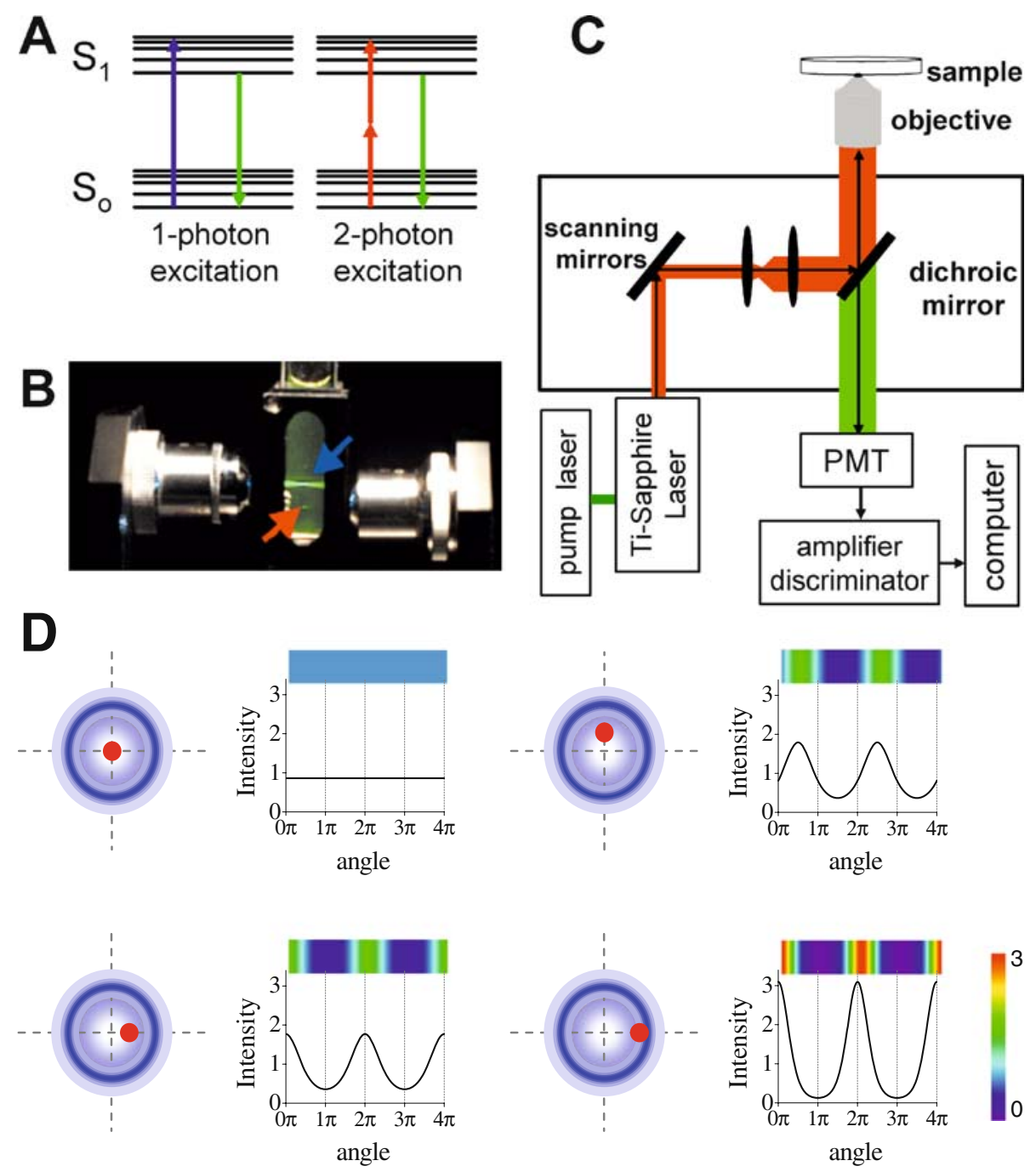

Figure 2. Two-photon microscopy. (A) Energy diagrams showing the electronic transitions occurring during one-photon (blue) and twophoton (red) excitation. (B) Emission volumes obtained in a fluorescein solution excited under one-photon (blue arrow) and two-photon (red arrow) conditions. The numerical apertures of the objectives used in this experiment were the same. (C) Scheme of the two-photon excitation microscope used for single-particle tracking experiments. (D) Intensity profile determined along two cycles of the tracking routine represented as a function of the angle of rotation of the laser, for different relative positions of the particle (black dot) with respect to the center of scanning (right panels). The laser orbits are represented with blue circles. Reprinted with permission from Levi et al. (2005b), copyright the Biophysical Society, Figure 2B is reprinted from So et al. (2000), with permission, from the Annual Review of Biomedical Engineering, Volume 2 (C) 2000 by Annual Reviews www.annualreviews.org.

achieve significant levels of two-photon excitation. This condition is fulfilled by using, for example, femtosecond-pulse lasers that provide pulses of high intensity during brief periods of time but have low average excitation power, therefore limiting the photodamage to the sample for at least two reasons: firstly, because the excitation of the fluorophore occurs only at the focal point of the laser beam and, secondly, because the near-IR laser light has relatively low absorption in biological samples.

Figure 2B shows the emission volume obtained in a sample of fluorescein under two-photon or onephoton excitation. While two-photon excitation occurs only in the femtoliter volume in which the laser was focused, one-photon excitation is also observed in out-of-focus regions. 
The key feature of two-photon microscopy is that emission occurs in a femtoliter focal volume. This small volume defines several advantages of this technique over one-photon excitation microscopy. It provides improved axial depth discrimination and image contrast and less out-of-focus photobleaching and photodamage since no excitation occurs in outof-focus regions. On the other hand, the background fluorescence in two-photon microscopy is usually lower because the excitation wavelength is significantly red-shifted with respect to emission. Thus, it is easier to separate excitation from emission lights than in a one-photon experiment where excitation and emission are spectrally close to each other. Another advantage of two-photon microscopy is that it is better suited for imaging thick specimens because the near-IR light used for the excitation is less scattered and absorbed than the UV or visible excitation light required for the excitation of common fluorophores in one-photon microscopy.

Figure 2D shows the two-photon microscope set-up used for the tracking experiments described below. In this set-up, a mode-locked titanium-sapphire laser is used as excitation source. These lasers are ideal for two-photon microscopy because they provide femtosecond pulses with a repetition rate of $\sim 100 \mathrm{MHz}$ and they can be tuned in the range $700-1000 \mathrm{~nm}$ covering the excitation of common fluorescent probes including most of the variants of fluorescent proteins. There are several papers describing the determination of two-photon excitation cross-section for fluorescent probes (see, for example, Xu \& Webb 1996, Xu et al. 1996, Larson et al. 2003, Wokosin et al. 2004).

The laser is then directed into the microscope by two galvomotor-driven scanning mirrors moved by voltage generated in a computer card. In a conventional two-photon microscope, the mirrors are programmed to raster scan the laser on a square region of the sample.

The laser light is reflected in a low-pass dichroic mirror and focused on the sample with the objective. Fluorescence emission is collected by the objective and passes through the dichroic mirror and a shortpass filter to eliminate any reflected excitation light. It then exits the microscope to the photomultiplier detector; the output of which is further amplified and passed through a photon-counting discriminator, and finally photons are counted with a data acquisition card.

\section{Single-particle tracking in a two-photon microscope}

Motivated by the results described above regarding the high level of photodamage observed in chromatin studies using standard fluorescence microscopes, we designed a routine for particle tracking in a two-photon excitation set-up which, as indicated, provides improved photostability of the sample (Levi et al. 2005a).

The main limitation of scanning microscopes is that acquiring an image usually takes on the order of a second depending on the size of the image to be scanned, making any tracking routine based on imaging very slow. Thus, a completely different approach to imaging-based methods was followed by our group (Kis-Petikova \& Gratton 2004, Levi et al. 2005a,b) based on the early theoretical work of Enderlein (Enderlein 2000).

The tracking routine starts with a fast raster scan of an area of the sample that includes the particle of interest. Then, the user selects the particle to be tracked by clicking on top of its image. This directs the laser beam to the chosen particle and the computer saves these coordinates as the initial position for the tracking routine. During each cycle of tracking, the excitation beam traces a given number of circular orbits surrounding the particle of interest and the fluorescence intensity is integrated at different points along the orbit. Figure 2D represents the fluorescence intensity profile expected during these circular scans for different positions of the particle relative to the center of scanning. The dependence of the intensity profile on the particle position during circular scans was mathematically derived, providing a way to determine the coordinates of the particle (Kis-Petikova $\&$ Gratton 2004). The spatial resolution depends on the diameter of the orbit and can be as high as $1 \mathrm{~nm}$ when the diameter of the circle is equal to the radial waist of the point spread function (Kis-Petikova \& Gratton 2004).

During the tracking routine, the determination of the particle position is done on the fly by analyzing the fast Fourier transform of the intensity signal (Kis-Petikova \& Gratton 2004). Before the next cycle of tracking, the center of scanning is moved to the position determined for the particle in the previous cycle. Thus, the laser follows the particle in real time; in contrast to imaging-based methods in which the trajectory is recovered from recorded data. 
The method can also be used to do simultaneous two-particle tracking. The tracking routine starts on top of one of the particles; after a given number of cycles, the laser jumps to the position of the second particle, where it performs the same number of tracking cycles. Then, the center of scanning is moved to the new position determined previously for the first particle. Thus, the positions of the particles are recovered alternately. It should be noted that by doing this the temporal resolution of the method is reduced to one half.

Experimentally, we determined that the spatial and temporal resolutions of the method are $20 \mathrm{~nm}$ and 32 $\mathrm{ms}$, respectively. Also, we showed that the error of the position determination is inversely proportional to the square of the $\mathrm{S} / \mathrm{N}$ ratio, as was predicted (Kis-Petikova \& Gratton 2004), being approximately constant for $\mathrm{S} / \mathrm{N}>2$ (Levi et al. 2005a). Most methods based on image analysis work only for $\mathrm{S} / \mathrm{N}$ ratios higher than 4 (Cheezum et al. 2001).

The background noise - due to the detection of compounds different from the particle of interest - is usually a limit to the accuracy of the tracking when doing experiments in living cells. The theoretical accuracy of the particle position obtained by the scanning method does not change in the presence of a locally homogeneous background representing up to $10 \%$ of the total photons emitted by the particle. In contrast, the theoretical accuracy of the particle position obtained by Gaussian deconvolution decreases abruptly with the addition of a similar background (Levi \& Gratton 2007).

\section{Tracking chromatin in a two-photon excitation microscope}

We re-inspected the motion of EGFP-tagged chromatin sequences in interphase cells with the twophoton microscopy tracking technique described above (Levi et al. 2005b). As mentioned previously, two-photon excitation usually causes less out-offocus photobleaching and photodamage than onephoton excitation. In addition, the excitation laser moves in a very small volume of the cell during the tracking routine, introducing no damage in regions far from the tagged sequence.

For this study, we used the C6-14 CHO cell line, which has 10-20 copies of a plasmid containing lac operator repeats and a DHFR cDNA transgene driven by a viral promoter (Tumbar \& Belmont 2001). These copies are integrated at a single, internal chromosome site and can be observed in the two-photon excitation microscope as a bright dot (Figure 3A).

The trajectories obtained for this EGFP-tagged sequence showed long periods of confinement in regions of sizes similar to the $30 \mathrm{~nm}$ fiber of chromatin-probably reflecting local, thermal fluctuations - interspersed with short periods in which the sequence moves $\sim 150 \mathrm{~nm}$ following a curvilinear path (Figure 3B).

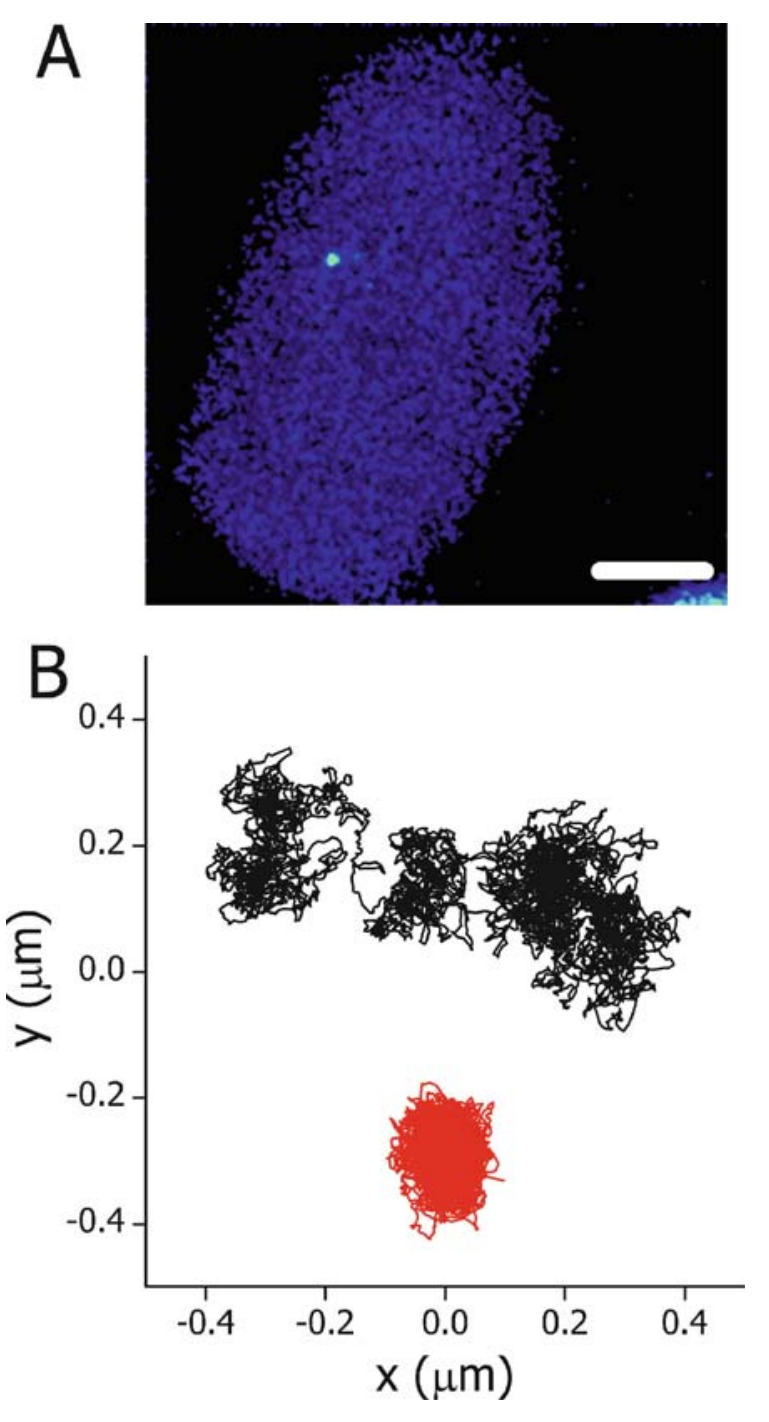

Figure 3. Dynamic EGFP-tagged sequence in living nuclei. (A) Two-photon excitation fluorescence image of a representative C6-14 cell. Bar $=5 \mu \mathrm{m}$. (B) Trajectories of the sequence in control (black) and azide-treated (red) cells. 
We were puzzled by the presence of these jumps in position since; as far as we know, they were not observed in previous studies of chromatin motion. However, jumps occurred in the time range $0.3-2 \mathrm{~s}$ and thus could not be observed using a tracking method with low temporal resolution. Moreover, the characteristic jump distance was $150 \mathrm{~nm}$, similar to the spatial resolution of previous methods.

We ruled out that jumps were due to the motion of the nucleus using the DHFR-BAC cell line, which has multiple lac operator-repeat insertions and also expressed the EGFP-lac repressor protein. Each of the insertions is visualized in a fluorescence microscope as a bright dot separated from the neighboring dots by less than 1-2 $\mu \mathrm{m}$. The trajectories of these labeled DNA sequences also presented jumps but they occurred independently from each other, showing that the jumps reflect local, short-distance motion of the chromatin sequence (Figure 4).

The way the sequence moves during jumps may remind us of the motion of a particle during active transport. However, in order to interpret the data properly, it is extremely important to do a careful statistical analysis of the data. A visual examination of isolated segments of any trajectory would suggest

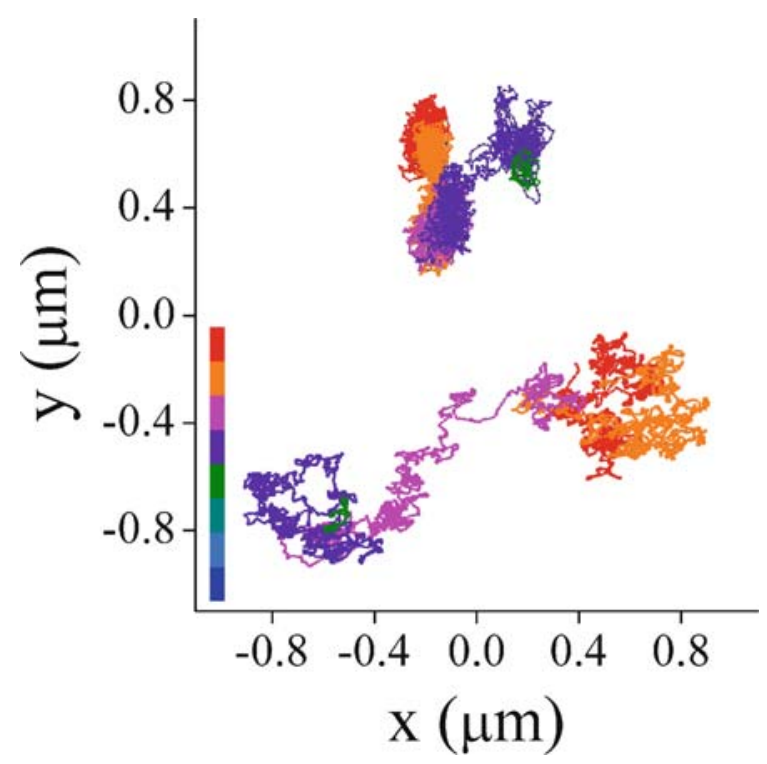

Figure 4. Simultaneous tracking of EFGP-tagged sequences in DHFR-BAC cells. The trajectories were registered by simultaneous tracking of two labeled sequences in a DHFR-BAC nucleus following the procedure described in the text. The color code represents the time evolution of the trajectories. different mechanisms of motion since there is always a non-zero probability of observing a given path in any trajectory. Unfortunately, most of the tools designed for trajectory analysis are based on MSD analysis, in which processes that occur only during short periods of time are averaged out.

We have therefore designed new statistical tools to gain insight into the mechanism underlying the motion of the sequence during jumps and have complemented this analysis with experiments to test the hypothesis derived from the statistical analysis. The statistical analyses were designed to compare the motion of the sequence during jumps with the predictions obtained assuming a passive diffusion process. All our analyses indicated that the sequence moves on average four times faster than in the periods between jumps and in paths more rectilinear than predicted for random diffusion motion, suggesting that an active process is responsible for the transport of the sequence during short periods of time. This hypothesis was supported by experiments showing, for example, that there are no jumps in the trajectories after ATP depletion (Figure 3B) suggesting that jumps likely reflect energy-dependent chromatin movements.

\section{Final remarks}

The view of the internal structure and function of the nucleus has changed drastically in recent years. It is now accepted that the nucleus is a well-organized and highly compartmentalized organelle. Chromatin has also been shown to be structurally organized in interphase and its organization was found to be very important to gene regulation. Significant advances in our understanding of chromatin dynamics during interphase were consequences of technological developments in the fields of fluorescence microscopy and cell biology. SPT constituted an important new tool to reveal new aspects of chromatin dynamics.

The emerging model coming from SPT experiments is that chromatin diffuses within a constrained volume of the nucleus most of the time but can also move through an energy-dependent mechanism during brief periods. Important new data from Chuang and colleagues (Chuang et al. 2006) gave some clues regarding active chromatin motion. They observed a fast, directional motion of an interphase chromosome 
site from the nuclear periphery to the interior after targeting a transcriptional activator to this site. The directional motion was suppressed in actin and myosin mutants, suggesting that they constitute the transport system required for this repositioning. More work will be needed to fully understand the mechanism involved in the fast, active motion of chromatin and, more importantly, the relevance of this motion to gene regulation.

\section{References}

Abramowitz M (2003) Microscope: Basics and Beyond. New York: Olympus America Inc.

Abranches R, Beven AF, Aragon-Alcaide L, Shaw PJ (1998) Transcription sites are not correlated with chromosome territories in wheat nuclei. J Cell Biol 143: 5-12.

Andrulis ED, Neiman AM, Zappulla DC, Sternglanz R (1998) Perinuclear localization of chromatin facilitates transcriptional silencing. Nature 394: 592-595.

Belmont AS, Li G, Sudlow G, Robinett C (1999) Visualization of large-scale chromatin structure and dynamics using the lac operator/lac repressor reporter system. Methods Cell Biol 58: 203-222.

Branco MR, Pombo A (2006) Intermingling of chromosome territories in interphase suggests role in translocations and transcription-dependent associations. PLoS Biol 4: e138.

Branco MR, Pombo A (2007) Chromosome organization: new facts, new models. Trends Cell Biol 17: 127-134.

Brickner JH, Walter P (2004) Gene recruitment of the activated INO1 locus to the nuclear membrane. PLoS Biol 2: e342.

Chambeyron S, Da Silva NR, Lawson KA, Bickmore WA (2005) Nuclear re-organisation of the Hoxb complex during mouse embryonic development. Development 132: 2215-2223.

Chaumeil J, Le Baccon P, Wutz A, Heard E (2006) A novel role for Xist RNA in the formation of a repressive nuclear compartment into which genes are recruited when silenced. Genes Dev 20: 2223-2237.

Cheezum MK, Walker WF, Guilford WH (2001) Quantitative comparison of algorithms for tracking single fluorescent particles. Biophys J 81: 2378-2388.

Chuang CH, Carpenter AE, Fuchsova B, Johnson T, de Lanerolle P, Belmont AS (2006) Long-range directional movement of an interphase chromosome site. Curr Biol 16: 825-831.

Chubb JR, Boyle S, Perry P, Bickmore WA (2002) Chromatin motion is constrained by association with nuclear compartments in human cells. Curr Biol 12: 439-445.

Cremer T, Cremer C (2006a) Rise, fall and resurrection of chromosome territories: a historical perspective. Part I. The rise of chromosome territories. Eur J Histochem 50: 161-176.

Cremer T, Cremer C (2006b) Rise, fall and resurrection of chromosome territories: a historical perspective. Part II. Fall and resurrection of chromosome territories during the 1950s to 1980s. Part III. Chromosome territories and the functional nuclear architecture: experiments and models from the 1990s to the present. Eur J Histochem 50: 223-272.
Cremer T, Cremer C, Schneider T, Baumann H, Hens L, KirschVolders M (1982) Analysis of chromosome positions in the interphase nucleus of Chinese hamster cells by laser-UVmicroirradiation experiments. Hum Genet 62: 201-209.

Crissman HA, Hirons GT (1994) Staining of DNA in live and fixed cells. Methods Cell Biol 41: 195-209.

Dahan M, Levi S, Luccardini C, Rostaing P, Riveau B, Triller A (2003) Diffusion dynamics of glycine receptors revealed by single-quantum dot tracking. Science 302: 442-445.

Dekker J, Rippe K, Dekker M, Kleckner N (2002) Capturing chromosome conformation. Science 295: 1306-1311.

Denk W, Strickler JH, Webb WW (1990) Two-photon laser scanning fluorescence microscopy. Science 248: 73-76.

Dundr M, Misteli T (2001) Functional architecture in the cell nucleus. Biochem J 356: 297-310.

Enderlein J (2000) Tracking of fluorescent molecules diffusing within membranes. Appl Phys B Lasers Opt 71: 773-777.

Handwerger KE, Gall JG (2006) Subnuclear organelles: new insights into form and function. Trends Cell Biol 16: 19-26.

Heard E, Bickmore W (2007) The ins and outs of gene regulation and chromosome territory organisation. Curr Opin Cell Biol 19: 311-316.

Hediger F, Taddei A, Neumann FR, Gasser SM (2004) Methods for visualizing chromatin dynamics in living yeast. Methods Enzymol 375: 345-365.

Heun P, Laroche T, Shimada K, Furrer P, Gasser SM (2001) Chromosome dynamics in the yeast interphase nucleus. Science 294: 2181-2186.

Kanda T, Sullivan KF, Wahl GM (1998) Histone-GFP fusion protein enables sensitive analysis of chromosome dynamics in living mammalian cells. Curr Biol 8: 377-385.

Kis-Petikova K, Gratton E (2004) Distance measurement by circular scanning of the excitation beam in the two-photon microscope. Microsc Res Tech 63: 34-49.

Kurz A, Lampel S, Nickolenko JE et al. (1996) Active and inactive genes localize preferentially in the periphery of chromosome territories. J Cell Biol 135: 1195-1205.

Lakadamyali M, Rust MJ, Babcock HP, Zhuang X (2003) Visualizing infection of individual influenza viruses. Proc Natl Acad Sci U S A 100: 9280-9285.

Larson DR, Zipfel WR, Williams RM et al. (2003) Water-soluble quantum dots for multiphoton fluorescence imaging in vivo. Science 300: 1434-1436.

Levi V, Gratton E (2007) Exploring dynamics in living cells by tracking single particles. Cell Biochem Biophys 48: 1-15.

Levi V, Ruan Q, Gratton E (2005a) 3-D particle tracking in a twophoton microscope: application to the study of molecular dynamics in cells. Biophys $J$ 88: 2919-2928.

Levi V, Ruan Q, Plutz M, Belmont AS, Gratton E (2005b) Chromatin dynamics in interphase cells revealed by tracking in a two-photon excitation microscope. Biophys $J$ 89: 4275-4285.

Lichter P, Cremer T, Borden J, Manuelidis L, Ward DC (1988) Delineation of individual human chromosomes in metaphase and interphase cells by in situ suppression hybridization using recombinant DNA libraries. Hum Genet 80: 224-234.

Mahy NL, Perry PE, Gilchrist S, Baldock RA, Bickmore WA (2002) Spatial organization of active and inactive genes and noncoding DNA within chromosome territories. J Cell Biol 157: 579-589. 
Marshall WF, Straight A, Marko JF et al. (1997) Interphase chromosomes undergo constrained diffusional motion in living cells. Curr Biol 7: 930-939.

Menon BB, Sarma NJ, Pasula S et al. (2005) Reverse recruitment: the Nup84 nuclear pore subcomplex mediates Rap1/Gcr1/Gcr2 transcriptional activation. Proc Natl Acad Sci U S A 102: 5749-5754.

Qian H, Sheetz MP, Elson EL (1991) Single particle tracking. Analysis of diffusion and flow in two-dimensional systems. Biophys J 60: 910-921.

Robinett CC, Straight A, Li G et al. (1996) In vivo localization of DNA sequences and visualization of large-scale chromatin organization using lac operator/repressor recognition. $J$ Cell Biol 135: 1685-1700.

Rosa A, Maddocks JH, Neumann FR, Gasser SM, Stasiak A (2006) Measuring limits of telomere movement on nuclear envelope. Biophys J 90: L24-26.

Saxton MJ (1993) Lateral diffusion in an archipelago. Singleparticle diffusion. Biophys J 64: 1766-1780.

Saxton MJ (1994a) Anomalous diffusion due to obstacles: a Monte Carlo study. Biophys J 66: 394-401.

Saxton MJ (1994b) Single-particle tracking: models of directed transport. Biophys J 67: 2110-2119.

Saxton MJ (1995) Single-particle tracking: effects of corrals. Biophys J 69: 389-398.

Saxton MJ (1996) Anomalous diffusion due to binding: a Monte Carlo study. Biophys J 70: 1250-1262.

Saxton MJ (1997) Single-particle tracking: the distribution of diffusion coefficients. Biophys J 72: 1744-1753.

Saxton MJ, Jacobson K (1997) Single-particle tracking: applications to membrane dynamics. Annu Rev Biophys Biomol Struct 26: 373-399.

Schmid M, Arib G, Laemmli C, Nishikawa J, Durussel T, Laemmli UK (2006) Nup-PI: the nucleopore-promoter interaction of genes in yeast. Mol Cell 21: 379-391.

Simonis M, Klous P, Splinter E et al. (2006) Nuclear organization of active and inactive chromatin domains uncovered by chromosome conformation capture-on-chip (4C). Nat Genet 38: $1348-1354$.
So PT, Dong CY, Masters BR, Berland KM (2000) Two-photon excitation fluorescence microscopy. Аnnu Rev Biomed Eng 2: 399-429.

Svoboda K, Yasuda R (2006) Principles of two-photon excitation microscopy and its applications to neuroscience. Neuron 50: 823-839.

Thompson RE, Larson DR, Webb WW (2002) Precise nanometer localization analysis for individual fluorescent probes. Biophys $J$ 82: $2775-2783$.

Tumbar T, Belmont AS (2001) Interphase movements of a DNA chromosome region modulated by VP16 transcriptional activator. Nat Cell Biol 3: 134-139.

Vazquez J, Belmont AS, Sedat JW (2001) Multiple regimes of constrained chromosome motion are regulated in the interphase Drosophila nucleus. Curr Biol 11: 1227-1239.

Verschure PJ, van Der Kraan I, Manders EM, van Driel R (1999) Spatial relationship between transcription sites and chromosome territories. J Cell Biol 147: 13-24.

Volpi EV, Chevret E, Jones T et al. (2000) Large-scale chromatin organization of the major histocompatibility complex and other regions of human chromosome 6 and its response to interferon in interphase nuclei. $J$ Cell Sci 113(Pt 9): $1565-1576$.

Wokosin DL, Loughrey CM, Smith GL (2004) Characterization of a range of fura dyes with two-photon excitation. Biophys $J \mathbf{8 6}$ : 1726-1738.

Xu C, Webb WW (1996) Measurement of two-photon excitaton cross sections of molecular fluorophores with data from $690 \mathrm{~nm}$ to $1050 \mathrm{~nm}$. J Opt Soc B 13: 481-491.

Xu C, Zipfel W, Shear JB, Williams RM, Webb WW (1996) Multiphoton fluorescence excitation: new spectral windows for biological nonlinear microscopy. Proc Natl Acad Sci U S A 93: 10763-10768.

Yildiz A, Forkey JN, McKinney SA, Ha T, Goldman YE, Selvin PR (2003) Myosin V walks hand-over-hand: single fluorophore imaging with 1.5-nm localization. Science 300: 2061-2065.

Zink D, Cremer T, Saffrich R et al. (1998) Structure and dynamics of human interphase chromosome territories in vivo. Hum Genet 102: 241-251. 